\title{
Processos Atencionais e Dependência-Independência de Campo: Estudo com Crianças e Adolescentes Portugueses
}

\author{
M. Adelina Guisande ${ }^{1}$ \\ Universidad de Santiago de Compostela, Espanha \\ Leandro S. Almeida \\ Universidade do Minho, Portugal \\ Filomena Ermida da Ponte \\ Universidade Católica Portuguesa, Portugal \\ Carolina Tinajero \\ Universidad de Santiago de Compostela, Espanha \\ M. Fernanda Páramo \\ Universidad de Santiago de Compostela, Espanha
}

\begin{abstract}
RESUMO - Este artigo relaciona o estilo cognitivo “dependência-independência de campo” com o desempenho em tarefas envolvendo vários processos e recursos de atenção. Com uma amostra de 98 crianças e 95 adolescentes portugueses, foram aplicadas quatro tarefas de atenção: capacidade de armazenamento (Dígitos em ordem direta); memória de trabalho verbal (Dígitos em ordem inversa); capacidade para dirigir, mudar e manter a atenção (Código); e capacidade de atenção sustentada (Teste de Atenção e Rastreio Visual, VSAT). Recorreu-se, ainda, à aplicação de uma prova de fator $g$, tendo em vista o controle da inteligência. Os resultados revelam diferenças entre dependentes e independentes de campo, especialmente relevantes no grupo de crianças na prova de VSAT. Esses resultados abrem novas linhas de investigação para explicar a pior execução acadêmica dos indivíduos dependentes de campo.
\end{abstract}

Palavras-chave: estilo cognitivo; dependência-independência de campo; processos de atenção.

\section{Attention Processes and Field Dependence-Independence: A Study with Portuguese Children and Adolescents}

\begin{abstract}
The present study relates the "field dependence-independence" cognitive style to the performance on tasks involving several attention processes and resources. With a 98 Portuguese children and 95 Portuguese adolescents sample, four attention tasks were applied: storage capacity (Digits Forward Test); verbal working memory (Digits Backward Test); capacity to focus, shift, and maintain attention (Digit Symbol Test); and capacity for sustained attention (Visual Search and Attention Test, VSAT). It was also applied a factor $g$ test for intelligence control. Results showed differences on attention processes between field-dependence and field-independence cognitive styles. These differences are more evident in the children subgroup on VSAT. These results open new research topics to explain lowest academic performance of field-dependent children.
\end{abstract}

Keywords: cognitive style; field dependence-independence; attention processes.

O estilo cognitivo dependência-independência de campo (DIC) é definido como a forma como as pessoas percebem os estímulos do ambiente e como organizam e codificam os aspetos específicos e gerais da informação nas suas situações de aprendizagem e de realização (Messick, 1984). Sujeitos independentes de campo tendem a ser mais analíticos no processamento da informação, conseguindo estar atentos aos pormenores e a elementos particularmente relevantes da informação a processar. Por sua vez, os sujeitos dependentes de campo recorrem mais frequentemente a uma abordagem global e menos analítica no processamento da informação. Os estudos sobre a relação entre DIC e o rendimento escolar (Chao, Huang \& Li, 2003; Kush, 1996; Tinajero \& Páramo, 1997; Zhang, 2004) apontam para uma superioridade nos resultados académicos por parte dos sujeitos independentes de campo (IC) em relação aos dependentes de campo (DC),

1 Endereço para correspondência: Departamento de Psicologia, Universidad de Santiago de Compostela, Campus Sur. 15782. Santiago de Compostela, Espanha.E-mail: mariaadelina.guisande@usc.es. generalizando-se essa superioridade às diversas áreas curriculares. Essa diferenciação tem justificado alguma investigação quanto aos processos cognitivos que diferenciam os sujeitos IC e DC, assim como sobre o impato diferenciado de ambos os estilos cognitivos no desempenho académico. Esses estudos pretendem, assim, ultrapassar a pouca validade interventiva ou o pouco valor informativo das avaliações por meio dos testes tradicionais de inteligência quando se pretende compreender como o aluno aprende e realiza na escola. Com efeito, os estilos cognitivos integram elementos mais dinâmicos e mais processuais da realização cognitiva, os quais podem contribuir, de uma forma mais clara, para explicar os bons e os fracos desempenhos académicos dos estudantes.

Uma das justificações para a relação entre a DIC e o rendimento académico dos alunos terá a ver com o funcionamento atencional dos sujeitos com diferentes estilos cognitivos (Davis \& Cochran, 1990; Guisande, Páramo, Tinajero \& Almeida, 2007). Três linhas de investigação, complementares 
entre si, emergiram na busca de uma explicação para esses resultados: uma primeira linha centra-se nas associações entre as provas de estilo cognitivo e as tarefas de vigilância; outra apoia-se nas diferenças entre sujeitos DC e IC nas tarefas de atenção selectiva; e, por fim, a terceira linha tenta clarificar as relações existentes entre o estilo cognitivo e a gestão do espaço atencional.

Com relação à primeira linha de investigação, os estudos relacionando tarefas de vigilância e a DIC apresentam resultados contraditórios. Alguns autores sugerem que os sujeitos IC são mais eficazes que os DC na deteção de sinais críticos, símbolos ou letras alvo (Amador \& Kirchner, 1999; Cahoon, 1970; Forbes \& Barrett, 1978; Moore \& Gross, 1973; Páramo, Corral, Rodríguez, Tinajero \& Cadaveira, 1999; Tinajero, Corral, Cadaveira \& Páramo, 1998), enquanto outros não encontraram essas diferenças (Fernández Ballesteros \& cols., 1980; Forns, Kirchner \& Amador, 1989; Kirchner, Forns \& Amador, 1990; Martínez Selva, 1987). Essas discrepâncias nos estudos complicam a interpretação da DIC no sentido dos processos atencionais associados à vigilância ou à deteção de elementos informativos, muito embora as discrepâncias observadas nos estudos se possam ter ficado a dever ao tipo de estimulação utilizada (modalidade sensorial, complexidade), à idade dos sujeitos e às próprias provas de estilo cognitivo usadas.

Em termos da segunda linha de investigação, existem investigações que analisaram a relação da DIC com a atenção seletiva por meio de dois parâmetros: aproximação à informação global versus analítica, e atenção a estímulos relevantes versus irrelevantes. Os resultados nesses estudos mostram uma maior congruência entre si. Os sujeitos DC centram-se preferencialmente nos aspectos globais da informação, o que suscita uma maior focalização em pistas irrelevantes ou distratoras (em tarefas auditivas ou visuais) que lhes dificultam a extração da informação relevante. Um padrão diferente no processamento cognitivo se encontra junto dos sujeitos IC. Esses resultados têm sido confirmados junto de crianças (Guisande, Tinajero, Rodríguez, Cadaveira \& Páramo, 2004; Ohlmann \& Carbonnel, 1983; Rozencwajg, 1991) e de adultos (Avolio, Alexander, Barrett \& Sterns, 1981; Burton, Moore \& Holmes, 1995; Clark \& Roof, 1988; Marendaz, 1985; Tsakanikos, 2006).

Por último, a terceira linha de investigação relaciona o estilo cognitivo com a memória de trabalho. Os autores assumem que a eficiência dos indivíduos no uso dos recursos da atenção irá condicionar o desempenho nas diversas tarefas cognitivas, como sejam, a capacidade de ler e ouvir, aquisição lexical e aprendizagem em geral, quer em crianças (Baillargeon, Pascual-Leone \& Roncadin, 1998; Guisande, Tinajero \& Páramo, 2005; Mansfield, 1997), adolescentes (Bahar \& Hansell, 2000; Mansfield, 1997; Páramo \& cols., 1999) ou adultos (Bennink, 1982; Cochran \& Davis, 1987; Goode, Goddard \& Pascual-Leone, 2002; Miyake, Witzki \& Emerson, 2001). De acordo com esses estudos, os indivíduos DC apresentam níveis inferiores de autorregulação e controle da sua realização cognitiva, explicando-se assim uma menor eficiência no uso dos seus recursos atencionais. Tomando as conclusões de um estudo recente (Guisande \& cols., 2007), as crianças IC e DC parecem não se diferenciar na capacidade de armazenamento de informação (memória a curto-prazo), diferindo, no entanto, no uso mais ou menos adequado das estratégias de controle e de distribuição do espaço atencional. Ao mesmo tempo, as crianças IC mostraram-se mais capazes na manutenção da atenção no processamento dos estímulos relevantes, apresentando ainda maior capacidade para dirigir a sua atenção consoante as exigências cognitivas colocadas por cada tarefa.

O presente estudo pretendeu verificar junto de crianças e adolescentes eventuais diferenças na utilização dos recursos atencionais em função do estilo cognitivo DC/IC que apresentam. Para essa análise, considera-se a avaliação de diferentes componentes da atenção, mais concretamente, a atenção seletiva, a atenção difusa, a atenção sustentada e o rastreio mental. Por outro lado, havendo estudos que apontam para alguma diferenciação nos resultados em testes de inteligência segundo o estilo cognitivo dos alunos, aplicou-se um teste de fator $g$ com o objetivo de isolar, nas análises estatísticas, o impacto específico da DIC nos processos atencionais.

\section{Método}

\section{Participantes}

A amostra (193 alunos) integra dois subgrupos de alunos portugueses com dois níveis etários: 98 crianças com idades compreendidas entre os 8 e os 9 anos de idade $(M=8,5)$, alunos do $3^{\circ}$ e $4^{\circ}$ anos de escolaridade; e 95 adolescentes com idades entre os 13 e os 14 anos $(M=13,5)$, do $7^{\circ}$ e $8^{\circ}$ anos. Todos esses alunos frequentavam uma escola privada da cidade de Braga, pertencendo a um estrato social médio e médio alto. Não se consideraram neste estudo crianças sinalizadas com dificuldades de aprendizagem, nomeadamente quando apoiadas pelas equipes da educação especial.

\section{Instrumentos}

Para avaliar o estilo cognitivo DIC dos adolescentes, utilizou-se o EFT - Embedded Figures Test (Witkin, Oltman, Raskin \& Karp, 1971). O teste consta de uma série de 24 lâminas com imagens complexas, nas quais o sujeito deve identificar uma figura simples, que se encontra escondida na imagem complexa. A pontuação é dada pelo número de figuras escondidas corretamente identificadas no tempo concedido (3 minutos). Uma pontuação mais elevada nesse teste indica uma maior independência de campo por parte do sujeito.

Para avaliar as crianças, utilizou-se o CEFT - Children Embedded Figures Test, uma versão decorrente do EFT (Karp \& Konstadt, 1971). Esse teste, de administração individual, está composto por 25 lâminas com imagens complexas e dois modelos recortados de figuras simples (triângulo e um pentágono irregular). Em cada figura complexa encontra-se escondida uma das formas simples que a criança deve localizar. $\mathrm{O}$ escore corresponde ao somatório dos elementos identificados corretamente. Nessa investigação foi considerado o tempo de realização da prova, concedendo-se o tempo máximo de 3 minutos para a resolução de cada item. À semelhança do EFT, uma pontuação mais elevada no CEFT é sinónimo de maior independência de campo. 
Para a avaliação do funcionamento atencional foram utilizados o Subteste de Dígitos da WISC-R (Wechsler, 1974/1998), o Subteste de Código da WISC-R e o Teste de Atenção e Rastreio Visual (VSAT) (Trenerry, Crosson, DeBoe \& Leber, 1990). O Subteste de Dígitos da WISC-R abrange duas partes: Dígitos de ordem direta e Dígitos de ordem inversa. Na primeira parte, Dígitos em ordem direta, o sujeito tem que repetir, respeitando a ordem de citação do experimentador, uma série de números progressivamente mais longa. O rendimento nessa tarefa indica, além da capacidade de armazenamento a curto prazo, um índice de resistência à distração. No segundo subteste, Dígitos de ordem inversa, pede-se ao sujeito que repita séries de números, mas na ordem inversa de apresentação. Assim, se obtém um índice da capacidade de rastreio mental e do espaço atencional do sujeito. Segundo vários autores, esse subteste é considerado uma medida da memória de trabalho verbal (Saito, 2001; Zelazo \& cols., 2003), associado aos processos atencionais e funções executivas (Gathercole \& Pickering, 2000; Hale, Hoeppner \& Fiorello, 2002; Hutton \& Towse, 2001), envolvendo também a transformação da informação, manipulação verbal e imaginação viso-espacial (Zhu \& Weiss, 2005).

O Subteste de Código da WISC-R consta de quatro filas de 25 quadrículas. Na parte superior de cada quadrícula, encontra-se um número distribuído aleatoriamente, de um a nove. A tarefa do sujeito consiste em copiar, o mais rapidamente possível, o símbolo correspondente a cada número. Essa tarefa tem um limite de tempo de 2 minutos, sendo a pontuação final dada pelo número de símbolos associados corretamente. Esse subteste é adequado à exploração da "atenção complexa”, uma vez que requer que o sujeito dirija, mude e mantenha a atenção (Lezak, 1995).

Por fim, o VSAT baseia-se na identificação, por parte do sujeito, de todas as letras ou símbolos iguais ao modelo apresentado e que se encontram intercaladas e distribuídas aleatoriamente. Para a identificação, o sujeito tem que destacar esses símbolos ou letras, assinalando-os com um traço. Essa prova compõe-se de quatro ensaios, sendo o primeiro e o segundo de treino. A tarefa tem um tempo limite de 60 segundos por cada ensaio e permite calcular uma pontuação direta baseada no número de acertos. Trata-se de um teste de procura visual, dirigido fundamentalmente a explorar o componente de vigilância ou atenção sustentada (Trenerry \& cols., 1990).

Finalmente, este estudo controlou a variável inteligência, assumindo-a como covariável nas análises estatísticas. Assim, para avaliar a inteligência (fator g) dos adolescentes, foi utilizada a Bateria de Provas de Raciocínio (BPR-5). Essa bateria avalia as capacidades de raciocínio, em termos indutivos e dedutivos (Almeida, 1992). A bateria é constituída por cinco provas. Contudo, este estudo recorreu apenas à prova de raciocínio abstrato, formada por 25 itens em formato de analogias (A:B - C:D) contendo figuras geométricas (similar aos testes de fator $g$ ). A pontuação na prova é dada através do somatório dos acertos, tendo o aluno à sua disposição cinco alternativas de resposta por item e usufruindo de 5 minutos para a realização da prova.

Em relação ao grupo de crianças, foi utilizada a prova de Matrizes Coloridas Progressivas de Raven (Coloured Progressive Matrices - CPM; Raven, 1947), referenciada como uma das melhores medidas psicológicas do fator $g$ (Sweetland \& Keyser, 1991) e estando validada para a população portuguesa (Simões, 1995). A prova é constituída por 36 itens organizados em três séries (A, Ab, B) de dificuldade cognitiva crescente, estando os 12 itens em cada uma das séries também organizados hierarquicamente por índice de dificuldade. A prova foi administrada de forma individual, sem limite de tempo. A pontuação corresponde ao total de exercícios corretamente respondidos.

\section{Procedimento}

A aplicação dos instrumentos foi precedida de um pedido de autorização aos órgãos diretivos e à associação de pais da escola. Uma vez obtido o seu consentimento, o segundo passo constou de um pedido formal às famílias dos alunos. Para tal, enviamos uma carta redigida pela diretora pedagógica, assegurando garantias suficientes de profissionalismo e rigor na investigação, uma carta onde se explicava a natureza e interesse do estudo, e um caderno de registro para preenchimento, por ambos os progenitores, de dados de identificação, nível de estudos e situação profissional, entre outros.

A aplicação das provas ocorreu em espaço escolar num horário acordado com a escola, assegurando o normal funcionamento das actividades escolares. A avaliação foi feita por um único avaliador, numa sessão única, com uma duração aproximada de 3 horas (incluindo alguns tempos de intervalo). A sessão iniciava-se com a explicação minuciosa a cada criança ou adolescente dos pormenores da avaliação.

\section{Análise de dados}

A amostra foi distribuída em três subgrupos de alunos: dependentes de campo (DC), intermédio e independentes de campo (IC), segundo a sua posição relativa em cada faixa etária $(8,9,13$ e 14 anos), de tal forma que os $33,3 \%$ das pontuações superiores no CEFT (maior número de respostas corretas) e no EFT (menor tempo de realização) foram consideradas como IC, os $33,3 \%$ das pontuações inferiores como DC, e os 33,3\% restantes, como grupo intermédio. De frisar que essa distribuição dos alunos pelos três subgrupos de estilo cognitivo foi feita dentro de cada idade (Tabela 1).

Tabela 1. Descrição dos participantes no estudo.

\begin{tabular}{cccccc}
\hline & \multicolumn{5}{c}{$\begin{array}{c}\text { Dependência-independência } \\
\text { de campo }\end{array}$} \\
\cline { 3 - 5 } & & DC & Intermédio & IC & Total \\
\hline Idade & 8 & 16 & 17 & 18 & 51 \\
& 9 & 15 & 17 & 15 & 47 \\
& 13 & 15 & 15 & 15 & 45 \\
& 14 & 17 & 17 & 16 & 50 \\
& & 63 & 66 & 64 & 193 \\
\hline
\end{tabular}


Foram também realizadas uma série de análises prévias com o objetivo de estudar a relação de algumas variáveis pessoais dos alunos (género, estatuto sócio-económico e inteligência) com a DIC e de indagar a necessidade do seu controle nas análises estatísticas posteriores em virtude do seu possível efeito no estilo cognitivo. Somente a variável inteligência apresentou uma associação estatisticamente significativa com os resultados na DIC, seja no teste de Matrizes Progressivas de Raven $\left[F_{(2,97)}=5,604 ; p<0,01\right]$, seja na Prova de Raciocínio Abstrato $\left[{ }_{(2,92)}=16,552 ; p<0,001\right]$, passando a ser considerada como covariável no desenrolar deste estudo. Uma vez que não se verificaram diferenças na DIC segundo o gênero [crianças $\left(\chi^{2}=1,036\right.$; gl=2; $\left.p=0,596\right)$; adolescentes $\left.\left(\chi^{2}=3,24 ; \mathrm{gl}=2 ; p=0,148\right)\right]$, as habilitações académicas [crianças $\left(\chi^{2}=5,393 ; \mathrm{gl}=2 ; p=0,067\right)$; adolescentes $\left.\left(\chi^{2}=1,964 ; \mathrm{gl}=2 ; p=0,375\right)\right]$ e o tipo de profissão da família [crianças $\left(\chi^{2}=1,301 ; \mathrm{gl}=2 ; p=0,522\right)$; adolescentes $\left(\chi^{2}=0,74\right.$; $\mathrm{gl}=2 ; p=0,964)$ ], essas variáveis não foram consideradas nas análises posteriores.

As análises dos resultados foram realizadas por meio do Programa SPSS (Versão 16.5 para Windows). Na comparação dos desempenhos dos três grupos de alunos em função do seu estilo cognitivo recorreu-se à análise de covariância (ANCOVA), controlando-se o score no teste de inteligência.

\section{Resultados}

Nas tabelas 2, 3 e 4 estão indicadas as médias e desviospadrão dos resultados nas tarefas de atenção tomando os alunos classificados segundo o estilo cognitivo. Na Tabela 2, verifica-se uma diminuição nas médias quando se passa dos Dígitos de ordem direta (capacidade de armazenamento) para os Dígitos de ordem inversa (memória de trabalho verbal), assim como um aumento esperado nas médias quando se passa do grupo de crianças para o grupo de adolescentes. Mediante as análises de covariância, não se verificou uma diferença estatisticamente significativa entre os três grupos de estilos cognitivos no Subteste de Dígitos em ordem direta, seja nos alunos de 8-9 anos $\left[F_{(1,97)}=0,012 ; p=0,915\right]$, seja nos alunos de 13-14 anos $\left[F_{(1,94)}=0,654 ; p=0,421\right]$. Do mesmo modo, não se verifica uma diferença estatisticamente significativa tomando os alunos repartidos pelos três estilos cognitivos no Subteste de Dígitos na ordem inversa. Essa situação ocorre junto dos alunos mais novos $\left[F_{(2,97)}=1,473 ; p=0,234\right]$ e junto dos adolescentes $\left[F_{(2,94)}=1,704 ; p=0,188\right]$, ainda que a oscilação na pontuação entre os dois estilos extremos na DIC se situe em apenas 0,6 pontos nas crianças e atinja já 1,5 pontos nos adolescentes. Não introduzindo a covariável inteligência na análise de variância, os resultados na prova de Dígitos em ordem inversa apresentam-se estatisticamente diferenciados $\left[F_{(2,94)}=3,05 ; p=0,052\right]$, a favor dos adolescentes IC.

No Subteste de Código (capacidade para dirigir, mudar e manter a atenção), os resultados parecem sugerir uma diferença de desempenho dos alunos dependentes de campo face aos outros dois grupos de alunos, seja nas crianças, seja nos adolescentes (Tabela 3). A diferença nas médias, para os dois grupos etários, é desfavorável aos alunos dependentes de campo. No entanto, procedendo à análise de covariância dos resultados dos três grupos na
DIC em função dos dois escalões etários, verifica-se que essas diferenças constatadas nas médias não se apresentam estatisticamente significativas, quer junto dos alunos mais novos $\left[F_{(2,97)}=2,810 ; p=0,065\right]$, quer junto dos adolescentes $\left[F_{(2,94)}=0,765 ; p=0,468\right]$. No entanto, essa situação se altera quando a análise não considera o efeito da inteligência (covariável), tanto em crianças $\left[F_{(2,97)}=3,261 ; p<0,05\right]$ quanto em adolescentes $\left[F_{(2,94)}=4,705 ; p<0,05\right]$.

Finalmente, os resultados dos alunos no VSAT (atenção sustentada), mostrados na Tabela 4, sugerem uma melhoria progressiva na passagem dos alunos DC para os alunos IC, sendo essa diferença favorável aos alunos IC. Pela análise de covariância realizada, observa-se uma diferença estatisticamente significativa nos resultados junto do grupo das crianças, a favor dos alunos IC $\left[F_{(2,97)}=3,948 ; p<0,05\right]$. No grupo de adolescentes, as diferenças nas médias não atingiram significado estatístico, muito embora se tenham aproximado desse nível crítico $\left[F_{(2,94)}=2,910 ; p=0,06\right]$. Quanto ao grupo de alunos mais novos, as comparações post-hoc apontam para uma diferença estatisticamente significativa entre os dois grupos de alunos mais contrastados em termos de estilo cognitivo (diferença de média de 9,2 pontos; $p<0,05)$, a favor dos alunos IC.

Tabela 2. Resultados no Subteste de Dígitos pelos três grupos de alunos.

\begin{tabular}{crrrrrrr}
\hline \multirow{2}{*}{ Teste } & \multirow{2}{*}{ Idade } & \multicolumn{2}{c}{ DC } & \multicolumn{3}{c}{ Intermédio } & \multicolumn{2}{c}{ IC } \\
\cline { 3 - 8 } & & $\mathbf{M}$ & $\mathbf{D P}$ & $\mathbf{M}$ & $\mathbf{D P}$ & $\mathbf{M}$ & $\mathbf{D P}$ \\
\hline Dígitos & $8-9$ & 8,2 & 2,33 & 8,0 & 2,15 & 8,1 & 2,37 \\
directos & $13-14$ & 11,3 & 1,96 & 11,3 & 1,81 & 11,6 & 1,59 \\
Dígitos & $8-9$ & 3,6 & 1,12 & 3,8 & 1,47 & 4,2 & 1,47 \\
inversos & $13-14$ & 7,2 & 2,76 & 7,7 & 2,41 & 8,7 & 2,41 \\
\hline
\end{tabular}

Tabela 3. Resultados no Subteste de Código pelos três grupos de alunos.

\begin{tabular}{ccccccccc}
\hline & & \multicolumn{2}{c}{ DC } & \multicolumn{3}{c}{ Intermédio } & \multicolumn{2}{c}{ IC } \\
\cline { 3 - 8 } Teste & Idade & \multicolumn{2}{c}{} & & & & & \\
\cline { 3 - 8 } & & M & DP & M & DP & M & DP \\
\hline \multirow{2}{*}{ Código } & $8-9$ & 33,1 & 6,80 & 36,3 & 5,52 & 36,3 & 4,92 \\
& $13-14$ & 60,6 & 9,55 & 67,0 & 11,30 & 67,1 & 8,05 \\
\hline
\end{tabular}

Tabela 4. Resultados na prova VSAT pelos três grupos de alunos.

\begin{tabular}{cccccccc}
\hline \multirow{2}{*}{ Teste } & \multirow{2}{*}{ Idade } & \multicolumn{2}{c}{ DC } & \multicolumn{2}{c}{ Intermédio } & \multicolumn{2}{c}{ IC } \\
\cline { 3 - 8 } & & $\mathbf{M}$ & $\mathbf{D P}$ & $\mathbf{M}$ & $\mathbf{D P}$ & $\mathbf{M}$ & $\mathbf{D P}$ \\
\hline \multirow{2}{*}{ VSAT } & $8-9$ & 68,2 & 12,45 & 70,3 & 12,98 & 77,4 & 11,79 \\
& $13-14$ & 92,0 & 13,40 & 102,5 & 20,27 & 103,4 & 13,48 \\
\hline
\end{tabular}




\section{Discussão}

O objetivo principal deste estudo foi analisar em que medida as crianças e os adolescentes dependentes e independentes de campo apresentam desempenhos diferenciados em tarefas que implicam diferentes processos atencionais. Os resultados obtidos sugerem a existência de diferenças atencionais em crianças e adolescentes em função do seu estilo cognitivo. Essas diferenças são especialmente evidentes no grupo de crianças, nas tarefas envolvendo atenção sustentada ou capacidade para manter a atenção nos estímulos relevantes. Nesse sentido, e segundo Huteau (1987), a forma como a informação ou os estímulos são apresentados aos sujeitos condiciona seu desempenho de acordo com o seu estilo cognitivo.

No sentido de outros dados disponíveis da investigação nessa área, as crianças dependentes de campo apresentaram um pior desempenho no teste de procura visual, dirigido fundamentalmente a avaliar o componente de vigilância ou atenção sustentada (VSAT). Esses resultados vão de encontro aos estudos que apontam uma associação estatisticamente significativa entre a DIC e a capacidade para manter a atenção sobre elementos ou partes da informação, ou a capacidade para se atender às pistas relevantes numa dada informação em presença de pistas distratoras (Avolio \& cols., 1981; Burton \& cols., 1995; Guisande \& cols., 2007).

Em segundo lugar, as crianças e adolescentes DC parecem aproveitar menos a sua capacidade atencional. Se forem observados seus desempenhos no Subteste de Dígitos na modalidade de evocação na ordem direta, a sua capacidade de retenção não é distinta da apresentada pelos seus colegas IC. No entanto, o seu menor desempenho nos Dígitos na modalidade de evocação na ordem inversa, sugere uma fraca utilização das estratégias de controle e de distribuição do espaço atencional. Já que para realizar uma busca na memória o sujeito tem que reorganizar a informação para libertar espaço de registro, esse processo parece estar menos eficiente nos dependentes de campo, dada a sua menor capacidade de reestruturação. Esses dados são corroborados por outros estudos com crianças, adolescentes e adultos (Baillargeon \& cols., 1998; Cochran \& Davis, 1987; Guisande \& cols., 2007; Johnstone \& Al-Naeme, 1991; Miyake \& cols., 2001; Niaz, 1989; Páramo \& cols., 1999; Saud, Rodríguez \& Niaz, 1993; Tinajero \& cols., 1998).

Numa lógica de aprofundar a compreensão das diferenças verificadas no espaço atencional, alguns autores avançam com leituras mais cognitivas tomando as funções ou processos mentais inerentes. Para Pascual-Leone (1997), a diferença encontrada parece não depender das diferenças da capacidade mental atencional, mas sim do recurso a esquemas executivos mais ou menos eficientes para mobilizar a dita capacidade. Por outro lado, com base no modelo da memória de trabalho (Baddeley \& Hitch, 1974), a variação individual na DIC poderá ser consequência das diferenças de eficiência no funcionamento do sistema de controle atencional em face da capacidade limitada do executivo central (uma das quatro estruturas que constitui o modelo da memória de trabalho). Assim, a utilização mais ou menos eficiente do executivo central poderá estar associada às várias subfunções realizadas na codificação, seguimento e supervisão da informação (Miyake \& cols., 2001; Niaz, 1989). O estilo cognitivo parece interferir nos desempenhos dos sujeitos, apresentando os DC mais dificuldades de uso de estratégias adequadas na resolução de tarefas quando as mesmas tarefas requerem maior complexidade de reestruturação da informação.

Em resumo, estes resultados podem assumir importantes implicações, tanto para a compreensão da natureza do estilo cognitivo, como para o planeamento do apoio educativo aos alunos ao longo da sua escolaridade. Se os alunos dependentes de campo experimentam dificuldades quando têm que extrair informação relevante e esta mesma informação não se encontra devidamente destacada, quando se requer centrar e manter a atenção numa tarefa complexa ou sustentada, ou quando enfrentam estímulos relevantes e irrelevantes de forma simultânea, logicamente esses alunos apresentam um pior rendimento nas tarefas escolares em que esses processos cognitivos são requisitos essenciais.

Nesse sentido, sugerimos duas possíveis direções para a investigação com o objetivo de potenciar o rendimento escolar dos alunos DC. Por um lado, importa saber como aumentar o seu ajustamento cognitivo e ultrapassar as suas dificuldades na aprendizagem por meio da aquisição, o mais precoce possível, de estratégias de processamento cognitivo não disponíveis no seu repertório habitual. Da mesma forma, importa investigar em que medida pode o professor tomar em consideração o estilo cognitivo dos seus alunos na planificação do seu ensino e na organização das atividades de aprendizagem em sala de aula. Face às preferências cognitivas dominantes nesses alunos, importa saber como os materiais didáticos, as formas de avaliação e os métodos de ensino usados pelo professor poderão melhor apoiar a sua aprendizagem e o seu sucesso escolar.

\section{Referências}

Almeida, L. S. (1992). Bateria de Provas de Raciocínio Diferencial (BPRD). Manual. Braga: Universidade do Minho.

Amador, J. A., \& Kirchner, T. (1999). Correlations among scores on measures of field dependence-independence cognitive style, cognitive ability, and sustained attention. Perceptual and Motor Skills, 88, 236-239.

Avolio, B. J., Alexander, R. A., Barrett, G. V., \& Sterns, H. L. (1981). Designing a measure of visual selective attention to assess individual differences in information processing. Applied Psychological Measurement, 5, 29-42.

Baddeley, A., \& Hitch, G. (1974). Working memory. Em G. H. Bower (Ed.), The psychology of learning and motivation: Advances in research and theory, Vol. 8 (pp. 47-89). New York: Academic Press.

Bahar, M., \& Hansel, M. H. (2000). The relationship between some psychological factors and their effect on the performance of grid questions and word association tests. Educational Psychology, 20, 349-364.

Baillargeon, R., Pascual-Leone, J., \& Roncadin, C. (1998). Mental-attentional capacity: Does cognitive style make a difference? Journal of Experimental Child Psychology, 70, 143-166.

Bennink, C. D. (1982). Individual differences in cognitive style, working memory, and semantic integration. Journal of Research in Personality, 16, 267-280. 
Burton, J. K., Moore, D. M., \& Holmes, G. A. (1995). Hypermedia concepts and research: An overview. Computers in Human Behavior, 11, 345-369.

Cahoon, R. (1970). Vigilance performance under hypoxia. Journal of Applied Psychology, 54, 479-483.

Chao, L., Huang, J., \& Li, A. (2003). A study of field independence versus field dependence of school teachers and university students in mathematics. Perceptual and Motor Skills, 97, 873-876.

Clark, H. T., \& Roof, K. D. (1988). Field dependence and strategy use. Perceptual and Motor Skills, 66, 303-307.

Cochran, K. F., \& Davis, J. K. (1987). Individual differences in inference processes. Journal of Research in Personality, 21, 197-210.

Davis, J. K., \& Cochran, K. F. (1990). An information processing view of field dependence-independence. Em O. N. Saracho (Ed.), Cognitive style and early education (pp. 61-78). New York: Gordon and Breach Science.

Fernández Ballesteros, R., Macia, A., Ruiz, J. M., Fernández Lagunilla, E., Del Villar, M. V., \& Díaz Vega, P. (1980). Influencia de la dependencia-independencia de campo sobre el efecto del feedback en una tarea de tiempos de reacción. Revista de Psicología General y Aplicada, 35, 589-595.

Forbes, J., \& Barrett, G. (1978). Individual abilities and task demands in relation to performance and satisfaction on two repetitive monitoring tasks. Journal of Applied Psychology, 63, 188-196.

Forns, M., Kirchner, T., \& Amador, J. A. (1989). Nivel de activación y variables de personalidad. Revista de Psicología General y Aplicada, 42, 469-473.

Gathercole, S. E., \& Pickering, S. J. (2000). Assessment of working memory in six-and seven-year-old children. Journal of Educational Psychology, 92, 377-390.

Goode, P. E., Goddard, P. H., \& Pascual-Leone, J. (2002). Event-related potentials index cognitive style differences during a serial-order recall task. International Journal of Psychophysiology, 43, 123-140.

Guisande, M. A., Páramo, M. F., Tinajero, C., \& Almeida, L. S. (2007). Field dependence-independence cognitive style: An analysis of attentional functioning. Psicothema, 19, 572-577.

Guisande, M. A., Tinajero, C., \& Páramo, M. F. (2005, agosto). Are there any factors that may influence the course of cognitive development? Working memory and field dependence-independence cognitive style. Painel apresentado na XII ${ }^{\text {th }}$ European Conference on Developmental Psychology, Tenerife, Espanha.

Guisande, M. A., Tinajero, C., Rodríguez, M. S., Cadaveira, F., \& Páramo, M. F. (2004). El estilo cognitivo dependenciaindependencia de campo y el uso de estrategias globales versus analíticas. Em F. Miras, N. Yuste \& F. Valls (Eds.), IV Congreso Internacional de Psicología y Educación: Calidad Educativa (pp. 2255-2261). Almería: Universidad de Almería.

Hale, J. B., Hoeppner, J. A. B., \& Fiorello, C. A. (2002). Analyzing digit span components for assessment of attention processes. Journal of Psychoeducational Assessment, 20, 128-143.

Huteau, M. (1987). Style cognitifet personalité. La dépendanceindépendance du champ. Lille: Presses Universitaires.

Hutton, U. M. Z., \& Towse, J. N. (2001). Short-term memory and working memory as indices of children's cognitive skills. Memory, 9, 383-394.
Johnstone, A. H., \& Al-Naeme, F. F. (1991). Room for scientific thought? Journal of Science Education, 13, 187-192.

Karp, S. A., \& Konstadt, N. L. (1971). The children's embedded figures test (CEFT). Em H. A. Witkin, P. K. Oltman, E. Raskin \& S. A. Karp (Eds.), A manual for embedded figures test (pp. 21-26). Palo Alto: Consulting Psychologist Press.

Kirchner, T., Forns, M., \& Amador, J. A. (1990). Relaciones entre las dimensiones de dependencia-independencia de campo, introversión-extroversión y tiempos de reacción. Anuario de Psicología, 46, 53-63.

Kush, J. C. (1996). Field-dependence, cognitive ability, and academic achievement in Anglo American and Mexican students. Journal of Cross-Cultural Psychology, 27, 561-575.

Lezak, S. (1995). Neuropsychological assessment (3a. ed.). New York: Oxford University Press.

Mansfield, E. A. (1997). Working memory development in adolescence: A neo-Piagetian investigation. Tese de Doutorado, University of Northern Colorado, Greeley.

Marendaz, C. (1985). Precedence globale et dependance du champ: Des routines visuelles? Cachiers de Psychologie Cognitive, 5, 727-745.

Martínez Selva, J. M. (1987). Locus de control, dependencia de campo y reflejo de orientación. Psicologemas, 1, 145-154.

Messick, S. (1984). The nature of cognitive styles: Problems and promise in educational practice. Educational Psychologist, 19, 59-74.

Miyake, A., Witzki, A., \& Emerson, M. (2001). Field dependence-independence from a working memory perspective: A dual-task investigation of the Hidden Figures Test. Memory, 9, 445-457.

Moore, S., \& Groos, S. (1973). Influence of critical signal regularity, stimulus event matrix, and cognitive style on vigilance performance. Journal of Experimental Psychology, 99, 137-139.

Niaz, M. (1989). Relation between Pascual-Leone's structural and functional M-space and its effects on problem solving in chemistry. International Journal of Science Education, 11, 93-99.

Ohlmann, T., \& Carbonnel, S. (1983). Dependance-independance a l'egard du champ et activites classificatoires sur objets significatifs. Em T. Ohlmann (Ed.), La pensée naturalle (pp. 275-285). Rouen: Presses Universitaires.

Páramo, M. F., Corral, M., Rodríguez, S., Tinajero, C., \& Cadaveira, F. (1999, julho). Cognitive functioning and field dependence-independence cognitive style. Painel apresentado na VI European Congress of Psychology, Rome, Itália.

Pascual-Leone, J. (1997). Constructivismo dialéctico como fundamento epistemológico de la ciencia humana. Revista Interamericana de Psicología, 31, 1-26.

Raven, J. C. (1947). Progressive matrices (Series A, Ab, B). London: H. K. Lewis.

Rozencwajg, P. (1991). Analysis of problem solving strategies on the Kohs Block Design Test. European Journal of Psychology of Education, 6, 73-88.

Saito, S. (2001). The phonological loop and memory for rhythms: An individual differences approach. Memory, 9, 313322.

Saud, G., Rodríguez, S., \& Niaz, M. (1993). Further evidence relating mental capacity, short-term storage space, and operational efficiency. Perceptual and Motor Skills, 76, 735-738. 
Simões, M. R. (1995). O Teste das Matrizes Progressivas Coloridas de Raven. Em L. S. Almeida, M. R. Simões \& M. Gonçalves (Eds.), Provas psicológicas em Portugal (pp. 221-252). Braga: APPORT.

Sweetland, R. C., \& Keyser, D. J. (Eds.) (1991). Tests: A comprehensive reference for assessments in psychology, education, and business ( $3^{\mathrm{a}}$ ed.). Austin: Pro-ed.

Tinajero, C., Corral, M., Cadaveira, F., \& Páramo, M. F. (1998, julho). Atención, percepción y estilo cognitivo en niños. Trabalho apresentado no II Congreso Iberoamericano de Psicología, Madrid, Espanha.

Tinajero, C., \& Páramo, M. F. (1997). Field dependenceindependence and academic achievement: A re-examination of their relationship. The British Journal of Educational Psychology, 67, 199-212.

Trenerry, M. R., Crosson, B., DeBoe, J., \& Leber, W. R. (1990). Visual Search and Attention Test. Odesa, FL: Psychological Assessment Resources.

Tsakanikos, E. (2006). Associative learning and perceptual style: Are associated events perceived analytically or as a whole? Personality and Individual Differences, 40, 579-586.

Wechsler, D. (1998). Escala de Inteligencia Wechsler para Niños-Revisada ( $4^{\mathrm{a}}$ ed.) Madrid: TEA. (Trabalho original publicado em 1974)
Witkin, H. A., Oltman, P. K., Raskin, E., \& Karp, S. A. (1971). A Manual for the Embedded Figures Tests. Palo Alto: Consulting Psychologists Press.

Zelazo, P. D., Muller, V., Frye, D., Marcovitch, S., Angitis, G., Boseovski, J., Chiang, J. K., Hongwanishkul, D., Schuster, B. V., \& Sutherland, A. (2003). The development of executive function in early childhood. Monographs of the Society for Research in Child Development, 68, 124-137.

Zhang, L. F. (2004). Field-dependence/independence: Cognitive style or perceptual ability? - Validating against thinking styles and academic achievement. Personality and Individual Differences, 37, 1295-1311.

Zhu, J., \& Weiss, L. (2005). The Wechsler Scales. Em D. P. Flanagan, J. L. Genshaft \& P. L. Harrison (Eds.), Contemporary intellectual assessment: Theories, tests and issues ( ${ }^{\text {nd }}$ ed.) (pp. 297-324). New York: Guilford.
Recebido em 16.01.08

Primeira decisão editorial em 01.09.08

Versão final em 10.09.08

Aceito em 19.09.08 